\title{
The Antidiabetic and Antioxidant Effects of Carotenoids: A Review
}

\author{
Miaad Sayahi ${ }^{1}$ and Saeed Shirali ${ }^{2,3^{*}}$ \\ 'Student Research Committee, Ahvaz Jundishapur University of Medical Sciences, Ahvaz, Iran \\ ${ }^{2}$ Hyperlipidemia Research Center, Department of Laboratory Sciences, Faculty of Paramedicine, \\ Ahvaz Jundishapur University of Medical Sciences, Ahvaz, Iran \\ ${ }^{3}$ Research Center of Thalassemia and Hemoglobinopathy, Ahvaz Jundishapur University of Medical Sciences, \\ Ahvaz, Iran; sayahi.m2014@gmail.com
}

\begin{abstract}
Carotenoids are a big group of phytochemicals that have a wide variety of protective and medical properties. They are widespread in plants and photosynthetic bacteria and have many medical functions. Here in this article, we studied antidiabetic and antioxidant effects of four kinds of carotenoids (lutein, lycopene, beta-carotene and astaxanthin) besides briefly defining them and also mentioned some of their plant sources. So, we can say, the aim of this study was to show some of the ways they can lower blood glucose and prevent the oxidant damages. Many articles, including originals and reviews were scanned in this way, but only a few had a suitable data. All of our references were articles has been collected electronically from valid journals and databases including PubMed, Science Direct, Elsevier, Springer and Google scholar. Beta-carotene is the most widely carotenoid in food prevent cancer and triggers the release of insulin and like lutein its antioxidant is useful for the prevention of macular degeneration. Lutein has also anticancer effects and reduces the ROS levels in the retina with diabetes. Lycopene helps to protect diabetes patients with cardiovascular disease. Astaxanthin has significant hypoglycemic effects. Both of lycopene and astaxanthin have powerful antioxidant activity. We suggest that each of these phytochemicals produces a kind of protect against diabetes and oxidative damages and also have other medical functions like anticancer, anti-inflammatory but further and deeper investigations are required in this field.
\end{abstract}

Keywords: Antidiabetic, Antioxidant, Astaxanthin, $\beta$-Carotene, Lutein, Lycopene, Phytochemical

\section{Introduction}

Reports from the World Health Organization (WHO) indicate that diabetes mellitus is one of the major killers of our time ${ }^{1}$. A long-term increase in glucose is one of the most important causes of diabetes secondary disorders such as angiopathy, neuropathy, retinopathy, deficiency in the antioxidant defense system, and lipid profile disorders ${ }^{2}$. Hyperglycemia alone does not cause diabetic complications. It is rather the detrimental effect of glucose toxicity due to chronic hyperglycemia, which is mediated and complicated through oxidative stress ${ }^{1}$. The single unifying mechanism of oxidative stress due to persistent hyperglycemia, which leads to an overt generation of ROS (Reactive Oxygen Species) in mitochondria, results in a variety of harmful oxidative products. Normalizing levels of mitochondrial, ROS have been shown to prevent the formation of these products ${ }^{3,4}$. Plants have been used for medicinal purposes across history and cultures and even across species. A majority of the world still relies heavily on herbal remedies for their primary health care. With the increasing movement of people across countries, there is an accompanying movement of their respective traditional medicines ${ }^{5}$. Phytochemicals are non-nutritive plant chemicals that have protective properties and have secondary metabolites in plants which provide much of the color and taste in fresh or processed fruits and vegetables. The plant produces these chemicals to protect itself, but recent research demonstrates that many phytochemicals can protect human against diseases ${ }^{6}$.

${ }^{*}$ Author for correspondence 
Carotenoids are widespread phytochemicals in plants and in photosynthetic bacteria, where they serve two essential functions: as accessory pigments in photosynthesis and in photoprotection. These two functions are a result of the conjugated polyene structure of carotenoids, which allows the molecule to absorb light and to quench, or inactivate, singlet oxygen and free radicals. Human, mostly consume a variety of different carotenoids, including those occurring naturally in foods (primarily fruits and vegetables) and those added as food colorants. Provitamin A carotenoids can be converted enzymatically in the intestinal mucosa to yield retinal and ultimately retinol; retinol (vitamin A) is required for vision, maintenance of differentiated epithelia, mucus secretion, and reproduction. Not all dietary carotenoids are metabolized in the intestinal mucosa following ingestion ${ }^{7}$. Numerous epidemiologic studies have shown that individuals who consume a relatively large quantity of carotenoid-rich fruits and vegetables have a decreased risk of cancer at several tumor sites, as reviewed elsewhere ${ }^{8}$. The mechanism by which carotenoids protect biological systems against $\mathrm{O}_{2}-$ mediated damage appears to depend largely on physical quenching 9 . Most carotenoids contain an extended system of conjugated double bonds, which is responsible for their antioxidant activity ${ }^{10}$. Carotenoids have also been shown to be capable of inhibiting free radical reactions ${ }^{11}$. The use of phytochemicals in the treatment of diseases is on the rise $e^{30-33}$. Also, determination of the contents of phytochemicals and plant extracts is important. Here, in this article we chose four carotenoids that have the most relationship with diabetes and antioxidant activities, including $\beta$-carotene, lycopene, lutein and astaxanthinand beside pointing out their bioactivities and therapeutic claims, tried to emphasize theirantidiabetic and antioxidant effects to show some of the ways they can lower blood glucose and prevent the antioxidant damages and also mentioned their herbal sources.

\section{B-Carotene}

Beta-carotene (BC) is a member of naturally occurring compounds called carotenoids which are a group of phytochemicals ${ }^{7,13}$. Beta-carotene is an antioxidant and is one of the forms of carotene that colored red-orange pigment and widely found in nature, abundant in plants and fruits, especially in orange fruits such as cantaloupe, mangoes, pumpkin, and papayas, and orange root vegetables such as carrots and sweet potatoes. The color of $\beta$-carotene is masked by chlorophyll in green leafy vegetables such as spinach, kale, and broccoli. It prevents a different type of cancers, by protecting cells in the body against oxidation damage by free radicals, by scooping them up and also by generating other antioxidants. What's more, it can also be converted to vitamin A as needed $^{12,13,34}$.

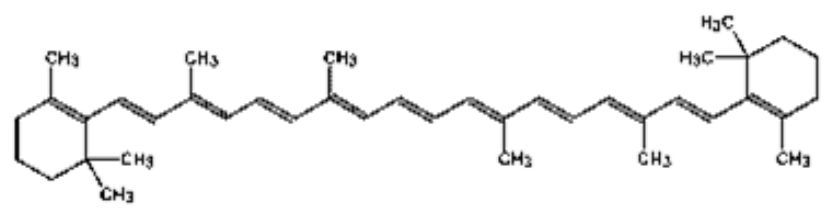

Figure 1. Chemical structure of $\beta$-carotene (MW: 536.84; Formula: $\left.\mathrm{C}_{40} \mathrm{H}_{56}\right)^{13}$.

Stanford University School of Medicine investigators has found that for people harboring a genetic predisposition that is prevalent among Americans, beta carotene, which the body converts to a close cousin of vitamin A, may lower the risk for the most common form of diabetes, while gamma tocopherol, the major form of vitamin $\mathrm{E}$ in the American diet, may increase risk for the disease. Moreover, the fact that both beta carotene and gamma tocopherol interact with the same gene variant to influence diabetes risk, albeit in opposite directions, suggests that the protein the gene called, SLC30A4, codes for may play a crucial role in the disease ${ }^{13,14}$. Indeed, that protein is relatively abundant in insulinproducing islet cells of the pancreas, where it aids the transport of zinc into those cells. This, in turn, triggers the release of insulin, whose adequate secretion by the pancreas and efficient uptake in muscle, liver, and fat tissue counters the dangerous buildup of glucose in the blood and, in the long run, the onset of type- 2 diabetes ${ }^{14}$. Antioxidants like $\mathrm{BC}$ seem to be useful for the prevention of macular degeneration and cataracts ${ }^{15}$. $\mathrm{BC}$ has potential antioxidant biological properties due to its chemical structure and interaction with biological membranes. It has been known for many years that carotenoids undergo "bleaching" i.e., lose their color when exposed to radicals or to oxidizing species. This process involves interruption of the conjugated doublebond system either by cleavage or by addition to one of the double bonds. Cleavage can be detected by characterizing the products that are formed, which are frequently carbonyls or epoxides ${ }^{16}$. In addition to the enzymatic cleavage of $\mathrm{BC}$ in mammalian metabolism, free radical attack on BC results in the formation of high amounts of cleavage products. For 
instance, $\beta$-apo-8'-carotenal and 6'-methyl- $\beta$-apo- 6 'carotene-6'-one (citranaxanthin) ${ }^{14}$.

\section{Lycopene}

Lycopene is a lipophilic compound with a 40 -carbon linear structure. Lycopene belongs to the family of fat-soluble plant pigments called carotenoids ${ }^{17}$. It is one of the major carotenoids found in tomatoes and tomato products. Unlike $\beta$-carotene it lacks a $\beta$-ionone ring, so it cannot be converted chemically to vitamin A. Lycopene is a bright red pigment that is naturally found in the human liver, serum (blood), adrenal glands, lungs, prostate, colon, and skin at higher levels than other similar pigments. Food processing may improve lycopene bioavailability and absorbance in two ways: (a) breaking down cell walls, which weakens the bonding forces between lycopene and the tissue matrix, thus making lycopene more accessible; and (b) enhancing the isomerization. After absorption of dietary fat, lycopene is transported via intestinal lymphatic to the liver by chylomicrons. Lycopene leaves the liver in a VLDL(very low-density lipoprotein) particle which is then catabolized to IDL(Intermediate Density Lipoprotein), the precursor of LDL. Under fasting conditions, lycopene circulates as part of LDL(low density lipoprotein). That is why usually a positive correlation exists between serum levels of lycopene and cholesterol ${ }^{17}$. A red pigment, lycopene is commonly found in fruits and vegetables of that hue. It may be the most abundant nutrient of the carotenoid family related to prostate health ${ }^{18}$. Over the last decade, there has been increased recognition that lycopene plays an important role in preventing the development of $\mathrm{CHD}$ (Coronary Heart Disease) and retarding the progression of atherosclerosis. In mice, lycopene has shown protection against the development of breast tumors ${ }^{19}$.

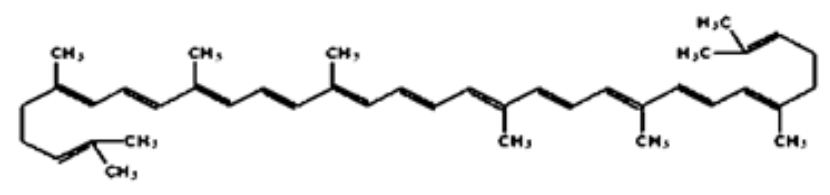

Figure 2. Chemical structure of lycopene (MW: 536.88; Formula: $\left.\mathrm{C}_{40} \mathrm{H}_{56}\right)^{13}$.

Diabetic patients may suffer from complications as vascular disease, diabetic neuropathies or infections. Lycopene helps to protect diabetes patients against cardiovascular disease and may improve the immune respons $\mathrm{e}^{13}$. Tomato paste, a tremendous source of this nutrient, has demonstrated some protection against the development of cardiovascular disease ${ }^{20}$. However, the consumption of lycopene seems not to reduce the risk of diabetes mellitus type $2^{13}$. Though not a pro-vitamin A, lycopene has attracted attention as a potent natural antioxidant. The antioxidant activity of lycopene is almost twice as high as that of $\beta$-carotene and has the greatest synergism with vitamin $\mathrm{E}^{17}$. This antioxidant activity of 13-carotene, which would be shared by other carotenoids as well because it depends on the formation of a resonancestabilized carbon-centered radical, may contribute to the protection of membranes from lipid peroxidation. Autoxidation of 13-carotene leads to the formation of epoxides located at the 13-ionone ring and to ketones (13-apocarotenones) and aldehydes (13-apo-carotenals) of different chain lengths. At low concentrations and at partial pressures of oxygen, such as those found in most tissues under physiologic conditions, 13-carotene was found to inhibit the oxidation of model compounds (tetralin and methyl linoleate) by peroxyl radicals ${ }^{11}$. Lycopene also shows benefit for the blood vessels around the heart, demonstrating protection to the blood vessels in the neck, better than vitamins A, E or CoQ10. This isn't much of a surprise since lycopene is regarded as a powerful antioxidant. Higher levels of this antioxidant have also shown protection against heart attack ${ }^{21}$. It is possible that anything red has some degree of lycopene, sure, but we should focus on incorporating tomato products regularly. Aside from the popular tomato, other sources of lycopene include red grapefruit, watermelon and apricots ${ }^{17}$.

\section{Lutein}

Lutein is an antioxidant carotenoid a pigmented nutrient that is responsible for the yellow colors of fruits and vegetables and is present in the highest quantities in dark, leafy green vegetables. You're born with a certain amount of lutein in your eye, but your body doesn't reproduce it. The macula is the region of the retina responsible for central vision. Lutein helps protect against this damage by filtering blue light before it can damage the macula ${ }^{22}$. It is related to beta-carotene and vitamin A. Lutein is one of two major carotenoids found as a color pigment in the human eye (macula and retina). Many people think of lutein as "the eye vitamin." They use it to prevent eye 
diseases, including Age-related Macular Degeneration (AMD), cataracts, and retinitis pigmentosa. Some people also use it for preventing colon cancer, breast cancer, type 2 diabetes, and heart disease ${ }^{23}$.

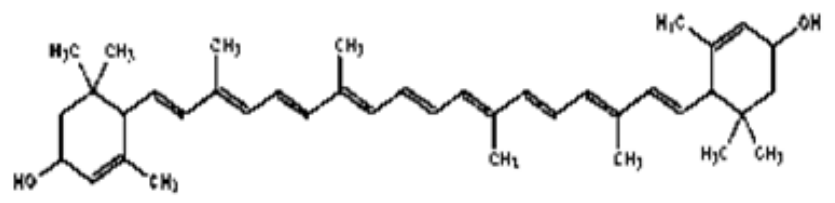

Figure 3. Chemical structure of lutein(MW: 568.87; Formula: $\left.\mathrm{C}_{40} \mathrm{H}_{52} \mathrm{O}_{2}\right)^{13}$.

Complications are a major fear of patients with diabetes, and these complications include cataracts, diabetic retinopathy, nephropathy, etc. Actually, it is thought that diabetes mellitus is associated with a fivefold higher prevalence of cataracts ${ }^{24}$. In one study it has been shown that lutein does not change the blood glucose level, but reduces the ROS level in the retina with diabetes. The effect of lutein in the retina was, at least in part, through the reduction of the diabetes-induced local ROS. Another influence of ROS-related to neuronal activity is exerted on the expression of brain-derived neurotrophic factor (BDNF).This factor regulates axonal growth and synaptic activity as well as neuronal survival. The level of BDNF in the diabetic retina is decreased, but lutein treatment prevents this reduction, protects the neuronal cells from apoptosis ${ }^{25}$. Of the many carotenoids circulating in the human serum, only lutein and zeaxanthin accumulated in the macular region of the retina, where they act as antioxidants. Lutein possesses pronounced free radical scavenging ability due to its polarity and number of conjugated double bounds ${ }^{26}$. The antioxidant, lutein, is presently being studied for its preventive effects on the progression of AMD, a vision-threatening disease, as a micronutrient supplement. However, interestingly, the preventive effect of lutein observed in the retina of the STZ-induced diabetes model occurs by the ROS reduction rather than the filtering of light energy. Moreover, the fact that lutein is physiologically delivered to the retinal neurons suggest that it might act directly on the retina, and not only as an antioxidant. Further studies aimed at elucidating whether lutein's effects involve pathways other than the antioxidative pathway should be performed ${ }^{25}$. Lutein and is a xanthophyll carotenoid found particularly in dark-green leafy vegetables and in egg yolks ${ }^{24}$. Foods rich in lutein include broccoli, spinach, kale, corn, orange pepper, kiwi fruit, grapes, orange juice, zucchini, and squash. Lutein is absorbed best when it is taken with a high-fat meal ${ }^{23}$.

\section{Astaxanthin}

Astaxanthin (3 ,30-dihydroxy-b, b-carotene-4, 40-dione), a keto-carotenoid pigment, with no pro-vitamin A activity ${ }^{27}$, is a carotenoid that has many highly potent pharmacological effects such as potent antioxidative activity, immunomodulating actions, anticancer activity, and anti-inflammation action ${ }^{28}$. Astaxanthin's pharmacological effects in animal models are extensive, ranging from protection against Helicobacter pyloriinduced gastric inflammation and the protection from hepatoxicity by carbon tetrachloride 13 to increase immune function ${ }^{29}$.

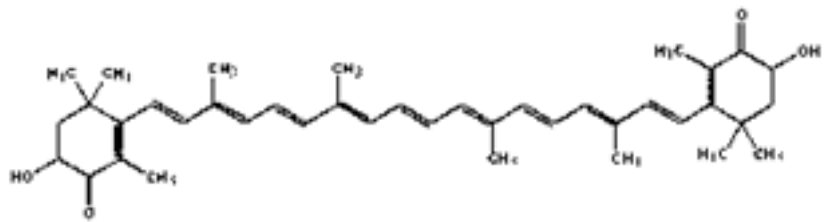

Figure 4. Chemical structure of astaxanthin (MW:596.84;Formula: $\left.\mathrm{C}_{40} \mathrm{H}_{52} \mathrm{O}_{4}\right)^{13}$.

In a study, results suggest that the astaxanthin from shrimp waste produces a significant hypoglycemic effect after oral administration in alloxan-induced diabetic mice. As presented in the "Result" the hypoglycemic effect of $10 \mathrm{mg} / \mathrm{kg}$ dose was to that of metformin. Postprandial hyperglycemia was significantly suppressed by oral administration of astaxanthin, which significantly lowered the postprandial AUC. Therefore, it can be said that astaxanthin is a useful natural agent in treating diabetes ${ }^{27}$. Astaxanthin is reported to be a potent antioxidant and inhibits low-density lipoprotein oxidation induced by azo-compound ${ }^{27}$. Astaxanthin has a high antioxidant activity due to the presence of the hydroxyl and keto endings on each ionone ring. It has been reported in the literature that the antioxidant activity of astaxanthin is approximately 10 times higher than other carotenoids such as zeaxanthin, lutein, canthxanthin, beta-carotene, and about 100-500 times than that of alfa-tocopherol ${ }^{27}$. Some findings suggest that astaxanthin may diminish the oxidative stress caused by hyperglycemia in the pancreatic $\beta$-cells ${ }^{29}$. 
Astaxanthin is a powerful biological antioxidant that occurs naturally in a wide range of organisms, including salmon, trout, shrimps, lobsters yeast, alga ${ }^{27}$, fish, and $\operatorname{birds}^{28}$.

\section{Conclusion}

As mentioned carotenoids are a big group of phytochemicals that have a wide variety of protective and medical properties. In this article, we studied antidiabetic and antioxidant effects of carotenoids including lutein, lycopene, beta-carotene, and astaxanthin. We suggest that each of these four phytochemicals has properties to protect against diabetes and oxidative damages and also have other medical functions like anticancer and anti-inflammatory activities but further and deeper investigations are required in this field.

\section{References}

1. Tiwari AK, Madhusudanarao J. Diabetes mellitus and multiple therapeutic approaches of phytochemicals: present status and future prospects. Current Science. 2002; 83(1):30-8.

2. Shirali S, Bathaie SZ, Nakhjavani M. Effect of crocin on the insulin resistance and lipid profile of streptozotocin-induced diabetic rats. Phytotherapy Research. 2013; 27(7):1042-7.

3. Brownlee $M$, Michael. Advanced protein glycosylation in diabetes and aging. Annual Review of Medicine. 1995; 46(1):223-34.

4. Nishikawa T, Edelstein D, Du XL, Yamagishi S-i, Matsumura T, Kaneda Y, et al. Normalizing mitochondrial superoxide production blocks three pathways of hyperglycaemic damage. Nature. 2000; 404(6779):787-90.

5. Pashazanousi MB, Raeesi M, Shirali S. Chemical composition of the essential oil, antibacterial and antioxidant activities, total phenolic and flavonoid evaluation of various extracts from leaves and fruit peels of Citrus limon. Asian Journal of Chemistry. 2012; 24(10):4331-4.

6. Dembinska-Kiec A, Mykkänen O, Kiec-Wilk B, Mykkänen H. Antioxidant phytochemicals against type 2 diabetes. British Journal of Nutrition. 2008; 99(E-S1):ES109-ES17.

7. Mayne ST. Beta-carotene, carotenoids, and disease prevention in humans. The FASEB Journal. 1996; 10(7):690-701.

8. Block G, Patterson B, Subar A. Fruit, vegetables, and cancer prevention: a review of the epidemiological evidence. $\mathrm{Nu}$ trition and Cancer. 1992; 18(1):1-29.

9. Krasnovsky A, Paramonova L. Interaction of singlet oxygen with carotenoids-rate constants of physical and chemical quenching. Biofizika. 1983; 28(5):725-9.
10. Comstock GW, Bush TL, Helzlsouer K. Serum retinol, beta-carotene, vitamin $\mathrm{E}$, and selenium as related to subsequent cancer of specific sites. American Journal of Epidemiology. 1992; 135(2):115-21.

11. Sies H, Stahl W. Vitamins E and C, beta-carotene, and other carotenoids as antioxidants. The American Journal of Clinical Nutrition. 1995; 62(6):1315S-21S.

12. van Arnum SD. Vitamin A. Kirk-Othmer Encyclopedia of Chemical Technology. 2000; 172-92. Doi: 10.1002/0471238 961.2209200101181421.a01.

13. Higuera-Ciapara I, Felix-Valenzuela L, Goycoolea FM. Astaxanthin: a review of its chemistry and applications. Critical Reviews in Food Science and Nutrition. 2006 Mar 1; 46(2):185-96.

14. Patel CJ, Chen R, Kodama K, Ioannidis JP, Butte AJ. Systematic identification of interaction effects between genome-and environment-wide associations in type 2 diabetes mellitus. Human Genetics. 2013; 132(5):495-508.

15. Agte V, Tarwadi K. The importance of nutrition in the prevention of ocular disease with special reference to cataract. Ophthalmic Research. 2010; 44(3):166-72.

16. Mueller L, Boehm V. Antioxidant activity of $\beta$-carotene compounds in different in-vitro assays. Molecules. 2011; 16(2):1055.

17. Watson RR, Preedy VR. Botanical medicine in clinical practice. Wallingford, UK: CABI; 2008.

18. Borek C. Antioxidants and the prevention of hormonally regulated cancer. Journal of Men's Health and Gender. 2005; 2(3):346-52.

19. Nagasawa H, Mitamura T, Sakamoto S, Yamamoto K. Effects of lycopene on spontaneous mammary tumour development in SHN virgin mice. Anticancer Research. 1994; 15(4):1173-8.

20. Sesso HD, Liu S, Gaziano JM, Buring JE. Dietary lycopene, tomato-based food products and cardiovascular disease in women. The Journal of Nutrition. 2003; 133(7):2336-41.

21. Kohlmeier L, Kark JD, Gomez-Gracia E, Martin BC, Steck SE, Kardinaal AF, et al. Lycopene and myocardial infarction risk in the EURAMIC Study. American Journal of Epidemiology. 1997; 146(8):618-26.

22. Zhu HX, Zheng JX. A review: structure, distribution, properties and physiological functions of lutein. China Food Additives. 2005; 5:011.

23. Kun G, Jing-Bo C, Mei L, Yu-Shun Z, Kai-Yan D. Study on oxidative degradation of lutein. Chemical Research and Application. 1999; 5:034.

24. Miranda M, Araiz J, Romero F. Lutein and Diabetic Cataracts. 2013.

25. Ozawa Y, Kurihara T, Sasaki M, Ban N, Yuki K, Kubota S, Tsubota K. Neural degeneration in the retina of the streptozotocin-induced type 1 diabetes model. Experimental Diabetes Research. 2011 Nov 17; 108328:7. Doi:10.1155/2011/108328.

26. Edakkadath RS, Korengath CP, Ramadasan K. Antioxidant activity of carotenoid lutein in vitro and in vivo. Indian Journal of Experimental Biology. 2010 Aug; 48(8): 843-8. 
27. Wang J-j, Chen Z-q, Lu W-q. Hypoglycemic effect of astaxanthin from shrimp waste in alloxan-induced diabetic mice. Medicinal Chemistry Research. 2012; 21(9):2363-7.

28. Naito Y, Uchiyama K, Aoi W, Hasegawa G, Nakamura N, Yoshida N, et al. Prevention of diabetic nephropathy by treatment with astaxanthin in diabetic $\mathrm{db} / \mathrm{db}$ mice. Biofactors. 2004; 20(1):49-59.

29. Uchiyama K, Naito Y, Hasegawa G, Nakamura N, Takahashi J, Yoshikawa T. Astaxanthin protects $\beta$-cells against glucose toxicity in diabetic $\mathrm{db} / \mathrm{db}$ mice. Redox Report. 2002; 7(5):290-3.

30. Ebrahimi E, Shirali S, Talaei R.The protective effect of marigold hydroalcoholic extract in STZ-induced diabetic rats: evaluation of cardiac and pancreatic biomarkers in the serum. Journal of Botany. 2016; 9803928:6. Doi:10.1155/2016/9803928.

31. Hosseini S, Gorjian M, Rasouli L, Shirali S. A comparison between the effect of green tea and kombucha prepared from green tea on the weight of diabetic rats. Biosci Biotechnol Res Asia. 2015; 20(1):141-5.

32. Ebrahimi E, Bahramzadeh S, Hashemitabar M, Mohammadzadeh G, Shirali S, Jodat J. Effect of hydroalcoholic leaves extract of Citrullus colocynthis on induction of insulin secretion from isolated rat islets of Langerhans. Asian Pac J Trop Dis. 2016; 6(8):638-41.

33. Shirali S, Babaali S, Babaali H. A comparative study on the effects of incretin and metformin on sugar profile and insulin resistance in STZ-induced diabetic Wistar rats. Res J Pharm Biol Chem Sci. 2016; 6(8):1921-29.

34. Kidmose U, Edelenbos M, Christensen LP, Hegelund E. Chromatographic determination of changes in pigments in spinach (Spinacia oleracea L.) during processing. J Chromatogr Sci. 2005; 46 (9):466-72. 